\title{
Mechanistic Insights Into the Zumach-Weiss-Kühle Synthesis of Dithiasuccinoyl (Dts)-Protected Amines
}

\author{
Matthew J. Henley, Michael J. Barany, Lin Chen, Robert P. Hammer, \\ Alex M. Schrader, Victor G. Young, Jr., and George Barany \\ Department of Chemistry, University of Minnesota, Minneapolis, MN, 55455, USA
}

\section{Introduction}

The 1,2,4-dithiazolidine-3,5-dione heterocycle was first prepared by Zumach, Weiss, and Kühle [1] by reactions of primary $O$-ethyl thiocarbamates with (chlorocarbonyl)sulfenyl chloride [Scheme 1]. Subsequently, we adopted this heterocycle as the basis for the thiolysable dithiasuccinoyl (Dts) amino protecting group, which was used for orthogonal solid-phase peptide synthesis and related applications [2-7]. We report here that when substrates are changed from primary amines to carbamates, cyclization to the title heterocycle does not occur, but two acyclic (chlorocarbonyl)disulfanyl species corresponding to proposed intermediates in the ZWK reaction mechanism can be isolated and structurally characterized. The present work might also provide insight into why reactions of primary amines with bis(chlorocarbonyl)disulfane fail to provide the corresponding Dts-amines, while analogous reactions with bis(trimethylsilyl)amines give Dts-amines in high yields [8].

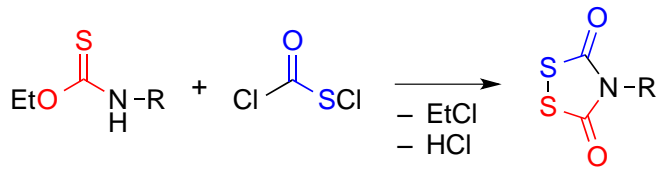

Scheme 1. Zumach-Weiss-Kühle (ZWK) reaction: Reaction of a primary thiocarbamate with (chlorocarbonyl)sulfenyl chloride results in the rapid formation of the 1,2,4-dithiazolidine-3,5-dione (Dts-amine) heterocycle, with simultaneous loss of ethyl chloride and hydrogen chloride.

\section{Results and Discussion}

In what was originally an attempt to prepare the Dts analogue of the $N$-phthaloylation reagent ( $N$-ethoxycarbonyl)phthalimide (i.e., the Nefkens reagent) [9] via ZWK chemistry, we discovered that the principal product was actually (chlorocarbonyl)( $N$-ethoxycarbonylcarbamoyl)disulfane [second box in Scheme 2], a surprisingly stabile crystalline compound. Through slight modifications to the reaction conditions, the precursor (chlorocarbonyl)[1-ethoxy-( $N$-ethoxycarbonyl)formimidoyl] disulfane [first box in Scheme 2] could be isolated and characterized as well. These two species correspond to proposed [1] intermediates in the ZWK mechanism that have never before been isolated or observed spectroscopically.

The two novel (chlorocarbonyl)disulfanyl species were characterized by their hydrolysis reactions, as well as by preparation of derivatives that substitute their respective chlorine atoms by methoxy and $\mathrm{N}$-methylaniline moieties through direct and/or indirect chemistries. Treatment of the (chlorocarbonyl)(carbamoyl)disulfane and its derivatives with base revealed that the diacyl $\mathrm{N}-\mathrm{H}$ is quite acidic, and that its abstraction leads to rapid decomposition of the disulfane.

The (chlorocarbonyl)(carbamoyl)disulfane, its methyl ester, and its $N$-methylanilide, along with the $N$-methylanilide of the precursor imidoyl disulfane, were all amenable to x-ray crystallography, laying the groundwork for detailed understanding of changing bond parameters in the ZWK reaction trajectory. As part of this analysis, we observed that for all four model disulfanes, the acidic proton or the lone pair electrons on the nitrogen are trans to the disulfane; this contrasts to the cis depiction in Scheme 2. A trans conformation will prevent the nucleophilic attack of nitrogen on the chlorocarbonyl moiety that is prerequisite to Dts formation. While we recognize that molecules may behave somewhat differently in solution, the rather stable and preferred trans conformation observed in the solid-state suggests a possible explanation why cyclization does not occur in the special case studied in the current work (Scheme 2). 


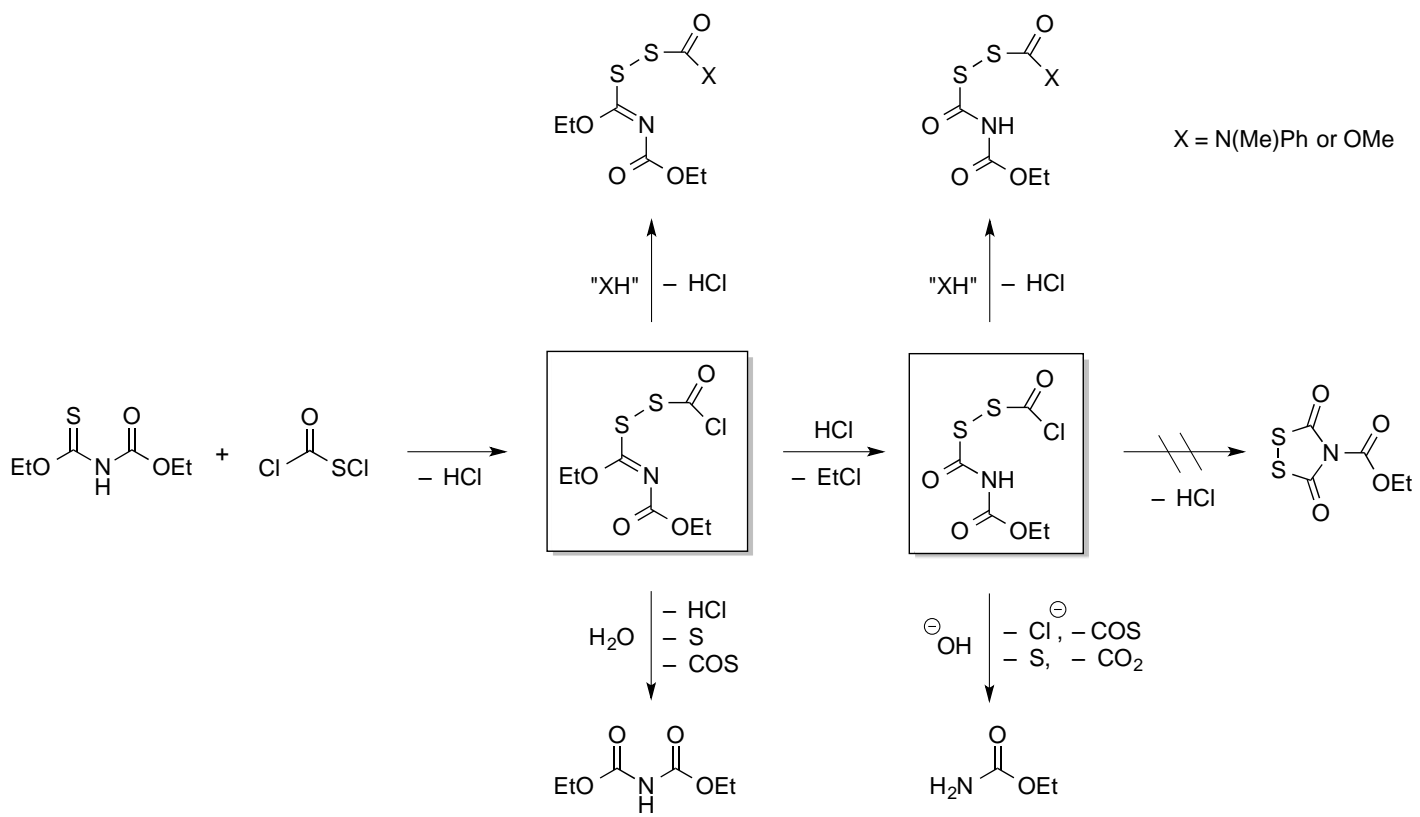

Scheme 2. Preparation and further transformations of two novel (chlorocarbonyl)disulfanyl species [shown within the two boxes]. Transformations in quotes indicate that the products were obtained either by direct substitution, or by indirect methods in certain cases when direct substitution reactions were not successful.

From the chemical point of view, we tentatively conclude that unsuccessful attempts at preparing Dtsamines directly from the reaction of bis(chlorocarbonyl)disulfane with primary amines failed at least partly because facile deprotonation of the putative (chlorocarbonyl)(carbamoyl) disulfane intermediate by any excess primary amine would promote degradation before any cyclization could occur.

\section{Acknowledgments}

MJH thanks Phylogica for sponsoring a travel award to present this work. VGY thanks NSF MRI grant 1229400, which provided funds for a Bruker PHOTON-100 diffractometer that was used to obtain some of the crystal structures presented in this work.

\section{References}

1. Zumach, G., Kühle, E. Angew Chem. Int. Ed. Engl. 9, 54-63 (1970), http://dx.doi.org/10.1002/anie.197000541

2. Barany, G., Merrifield, R.B. J. Am. Chem. Soc. 99, 7363-7365 (1977), http://dx.doi.org/10.1021/ja00464a050

3. Jensen, K.J., Hansen, P.R., Venugopal, D., Barany, G. J. Am. Chem. Soc. 118, 3148-3155 (1996), http://dx.doi.org/10.1021/ja953529i

4. Barany, G., Albericio, F. J. Am. Chem. Soc. 107, 4936-4942 (1985), http://dx.doi.org/10.1021/ja00303a019

5. Chen, L., Barany, G. Lett. Pept. Sci. 3, 283-292 (1996), http://dx.doi.org/10.1007/BF00127662

6. Xu, Q., Musier-Forsyth, K., Hammer, R.P., Barany, G. Nucleic Acids Res. 24, 1602-1607 (1996), http://dx.doi.org/10.1093/nar/24.9.1602

7. Planas, M., Bardají, E., Jensen, K.J., Barany, G. J. Org. Chem. 64, 7281-7289 (1999), http://dx.doi.org/10.1021/jo9824394

8. Barany, M.J., Hammer, R.P., Merrifield, R.B., Barany, G. J. Am. Chem. Soc. 127, 508-509 (2005), http://dx.doi.org/10.1021/ja0455446

9. Nefkens, G.H.L., Tesser, G.I., Nivard, R.J.F. Rec. Trav. Chim. Pays-Bays 79, 688-698 (1960), http://dx.doi.org/10.1002/recl.19600790705 